EPSC Abstracts

Vol.14, EPSC2020-234, 2020

Europlanet Science Congress 2020

(C) Author(s) 2020. This work is distributed under

the Creative Commons Attribution 4.0 License.

\title{
Using rover wheels to study regolith dynamics
}

Cecily Sunday ${ }^{1,2}$, Naomi Murdoch ${ }^{1}$, Simon Tardivel ${ }^{3}$, Nicola Imperatore ${ }^{1}$, Patrick Michel ${ }^{2}$, and Stephan Ulmec ${ }^{4}$

${ }^{1}$ Institut Supérieur de I'Aéronautique et de l'Espace, Department of Electronics, Optronics, and Signal Processing (DEOS),

Toulouse, France (cecily.sunday@isae-supaero.fr)

'Université Côte d'Azur, Observatoire de la Côte d'Azur, Centre National de la Recherche Scientifique (CNRS), Laboratoire Lagrange, Nice, France

${ }^{3}$ Centre National d'Études Spatiales (CNES), Toulouse, France

${ }^{4}$ Deutsches Zentrum für Luft- und Raumfahrt e.V. (DLR), Cologne, Germany

\section{Abstract}

In this study, we present several ways in which a rover wheel can be used as a tool to study regolith dynamics. We demonstrate specific analysis methods by conducting numerical simulations of a simple rover wheel traversing a bed of regolith. The simulation data is used to analyze the general flow behavior of granular material around a wheel in a reduced-gravity environment.

\section{Introduction}

In the mid-2020s, the Martian Moons eXploration mission (MMX) will visit Phobos and Deimos, the moons of Mars. The key science objectives of the Japanese mission are to clarify the origins of the Martian moons and to investigate the mechanisms behind the evolution of these bodies. During the MMX mission, the French Space Agency (CNES) and the German Aerospace Center (DLR) will deploy a small, $25 \mathrm{~kg}$, wheeled rover to the surface of Phobos [4,6]. Wheeled rovers have been used to explore the Moon and Mars, but they have never been used to traverse small-body surfaces. Like the Moon and Mars, Phobos is thought to be covered by a layer of loose regolith. Its low-gravity environment, however, is comparable to that of a large asteroid. As such, the MMX rover will serve primarily as a technology demonstration. It will be used to evaluate the performance of a wheeled locomotion system on a granular surface in milli-gravity conditions.

The MMX rover provides a unique opportunity to make in-situ observations on the surface of Phobos. Among other instruments, the rover will be equipped with two navigation cameras (NavCams) and two wheel cameras (WheelCams). The WheelCams will capture the direct interaction between the rover's wheels and surface of Phobos. When combined with images and engineering data, a rover's wheels can become powerful tools for studying the surface mechanical properties of soil. For instance, an extensive study on the cohesion and frictional properties of the Martian surface was conducted by observing trenches created by Spirit and Opportunity, the Mars Exploration Rovers (MER) [2]. In another example, the strength and stiffness properties of lunar soil were estimated using lander images and wheel sinkage estimates from China's Yutu rover on the Cheng'E-3 mission [1].

The objective of this work is to present different types of regolith-focused studies that can be made by treating a rover wheel as an excavation tool. We begin by providing a survey of techniques that 
have been employed in the past. Then, we demonstrate how numerical modeling can be used to augment in-situ observations from actual planetary missions.

\section{Simulations}

Wheel-regolith simulations are conducted using the soft-sphere DEM implementation in the opensource code Chrono $[3,5]$. For simplicity, we consider a generic wheel design that consists of a cylindrical hub and paddle-like grousers. The rover wheel is constructed in Chrono using two different methods. The first method consists of representing the wheel as an assembly of cylinders and boxes. In the second method, the wheel is imported into the simulations as a custom triangular mesh. Comparing the results for the two different wheel models helps to validate the meshing technique so that more complex wheel designs can be used in future simulations.

Preliminary regolith beds consist of approximately 150,000 mono-disperse spherical particles. The particles have material strength properties comparable to quartz sand and are settled in a rectangular container with random-loose packing. After the particles settle, the wheel is added to the simulations so that it sits just above the surface of the bed. We let the wheel sink into the granular material under some gravity level and then rotate the wheel at a low and constant speed. The wheel makes a quarter of a turn and is free to translate in the vertical and horizontal directions. Position, velocity, force, and torque information is reported for the wheel and all of the particles.

\section{Results}

Simulations are conducted for Earth and reduced-gravity levels and for varied magnitudes of particle cohesion. Preliminary results for wheel sinkage and slip are reported, with particular focus on the motion and behavior of the grains directly beneath the wheel. The simulation results are discussed in the larger context of how to use wheel-regolith modeling to augment surface-science experiments performed by actual planetary rovers.

\section{Acknowledgements}

We would like to thank ISAE-SUPAERO and the Centre National d'Études Spatiales (CNES) for financially supporting this research effort.

\section{References}

[1] Gao, Yang, et al. "Lunar soil strength estimation based on Chang'E-3 images." Advances in Space Research 58.9 (2016): 1893-1899.

[2] Sullivan, R, et al. "Cohesions, friction angles, and other physical properties of Martian regolith from Mars Exploration Rover wheel trenches and wheel scuffs." Journal of Geophysical Research: Planets 116.E2 (2011).

[3] Sunday, C., et al. "Validation of Chrono for granular DEM simulations in reduced-gravity environments." (2020) [submitted].

[4] Tardivel, S., et al. "The MMX rover: An innovative Design enabling Phobos in-situ Exploration." In LCPM Low-Cost Planetary Missions Conference, IAA, 2019.

[5] Tasora, Alessandro, et al. "Chrono: An open source multi-physics dynamics engine." International Conference on High Performance Computing in Science and Engineering, Springer, Cham , 2015.

[6] Ulamec, S., et al., "A rover for the JAXA MMX mission to Phobos." In 70th International Astronautical Congress (IAC), 2019. 